\title{
Development and Use of an Efficient DNA-Based Viral Gene Silencing Vector for Soybean
}

\author{
Chunquan Zhang, Chunling Yang, Steven A. Whitham, and John H. Hill \\ Department of Plant Pathology, lowa State University, Ames 50011, U.S.A.
}

Submitted 17 June 2008. Accepted 30 September 2008.

\begin{abstract}
Virus-induced gene silencing (VIGS) is increasingly being used as a reverse genetics tool to study functions of specific plant genes. It is especially useful for plants, such as soybean, that are recalcitrant to transformation. Previously, Bean pod mottle virus (BPMV) was shown to be an effective VIGS vector for soybean. However, the reported BPMV vector requires in vitro RNA transcription and inoculation, which is not reliable or amenable to high-throughput applications. To increase the efficiency of the BPMV vector for soybean functional genomics, a DNA-based version was developed. Reported here is the construction of a Cauliflower mosaic virus $35 \mathrm{~S}$ promoter-driven BPMV vector that is efficient for the study of soybean gene function. The selection of a mild rather than a severe BPMV strain greatly reduced the symptom interference caused by virus infection. The DNA-based BPMV vector was used to silence soybean homologues of genes involved in plant defense, translation, and the cytoskeleton in shoots and in roots. VIGS of the Actin gene resulted in reduced numbers of Soybean mosaic virus infection foci. The results demonstrate the utility of this new vector as an efficient tool for a wide range of applications for soybean functional genomics.
\end{abstract}

Additional keywords: Comovirus, Potyvirus

Forward genetics has successfully allowed the study of gene function in many plant species. Characteristically, it is associated with the cloning of a gene responsible for a mutant phenotype. Recently, the availability of whole genome sequences or large numbers of sequenced cDNA clones has provided new approaches to identify genes involved in particular biological functions. One of the most important new approaches is reverse genetics, in which the expression of a known gene or sequence is altered and the phenotype resulting from the altered gene expression is investigated. Recently, virus-induced gene silencing (VIGS), a transient reverse genetics tool for characterizing gene functions, has been widely used in many plants (Burch-Smith et al. 2004; Senthil-Kumar et al. 2008).

VIGS downregulates gene expression through RNA silencing that involves sequence-specific RNA degradation at the post-transcriptional level in plants. The transient nature of VIGS provides several advantages over other methods for associating genes with traits. VIGS overcomes the requirement for stable plant transformation which is time consuming and difficult for many plant species (Burch-Smith et al. 2004; Senthil-Kumar et

Corresponding author: Chunquan Zhang; Telephone: +1 (515) 294-3949; Fax: +1 (515) 294-9420; E-mail:czhan2@iastate.edu al. 2008). VIGS can also be used to target genes that, when stably mutated or silenced, cause lethal effects on plant development (Burch-Smith et al. 2006; Lu et al. 2003; Turnage et al. 2002). The increasing availability of genome databases enables design of VIGS to downregulate gene expression of a single gene or paralogous genes. Due to these inherent advantages, many plant viral gene-silencing vectors have recently been developed (Burch-Smith et al. 2004; Constantin et al. 2004; Ding et al. 2006; Senthil-Kumar et al. 2008). Among the many VIGS vectors that have been developed, some are designed to use prokaryotic promoters for in vitro RNA transcription to produce infectious viral genomic RNA for inoculation. Others utilize eukaryotic promoters that facilitate direct introduction of infectious cDNA clones into plants for inoculation. A key aspect of the design that affects efficiency is placement of the VIGS elements under control of a Cauliflower mosaic virus (CaMV) $35 \mathrm{~S}$ promoter and Nos terminator. DNAbased vectors provide distinct advantages over RNA-based vectors because cost and time are reduced and they are easier to manipulate and introduce into plants. The bypassing of RNA transcription and the DNA-based inoculation provides increased throughput. The latter strategy was employed for the Tobacco rattle virus (TRV) and Potato virus $X$ (PVX) vectors that have been successfully used for high-throughput screening for the study of gene function (Liu et al. 2002; Lu et al. 2003).

Soybean is a major oilseed crop and the main food protein source in the world. Because of its moderate genome size and rapidly accumulating genomic sequences, the United States Department of Agriculture and United States Department of Energy jointly initiated efforts to sequence the soybean genome (Jackson et al. 2006). VIGS was identified as a necessary functional genomics tool for soybean and high-throughput VIGS was regarded as an ideal reverse-genetics tool for large-scale soybean functional genomics aimed at translating the genomic information into functional knowledge (Jackson et al. 2006; Stacey et al. 2004).

Bean pod mottle virus (BPMV, genus Comovirus) has a bipartite positive-strand RNA genome consisting of RNA-1 (approximately $6.0 \mathrm{~kb}$ ) and RNA-2 (approximately $3.6 \mathrm{~kb}$ ). Previously, an in vitro RNA transcription-based BPMV VIGS vector was developed and shown to be useful for soybean gene silencing (Zhang and Ghabrial 2006). However, the inoculation efficiency of RNA transcripts limits the use of mild BPMV strains for soybean VIGS study; instead, a severe strain has been used for further study (Kachroo et al. 2008). In the work reported here, a DNA-based vector was developed with high infection efficiency and enhanced throughput by placing BPMV genomic elements under the control of a CaMV $35 \mathrm{~S}$ promoter and Nos terminator. This enables use of 
a mild BPMV strain for VIGS in soybean that can significantly reduce interference caused by severe leaf symptoms resulting from infection by the virus. The DNA-based BPMV vector was used to silence soybean homologues of genes involved in plant defense, translation, and the cytoskeleton. The potential for study of gene function was demonstrated by association of repressed expression of an Actin gene, a key component of the cytoskeleton, with a reduction in Soybean mosaic virus (SMV, genus Potyvirus) infection foci. In addition, the BPMV vector was also used to silence genes in the root. Thus, the results demonstrate the utility of this new vector as an efficient tool for a wide range of applications in soybean functional genomics.

\section{RESULTS}

\section{Infectivity of RNA-based BPMV VIGS vectors.}

Previously, soybean phytoene desaturase (PDS) and stearoyl-acyl carrier protein-desaturase were silenced using the RNA-based BPMV vector that requires in vitro RNA transcription and mechanical RNA inoculation (Kachroo et al. 2008; Zhang and Ghabrial 2006). In order to determine infectivity of the RNA-based VIGS vector, we selected soybean homologues of a variety of genes, including those involved in plant defense, translation, and the cytoskeleton, for VIGS. Seven single-gene VIGS constructs were developed using the RNA-based BPMV vector pGG7R2-V from BPMV strain KG7 (Tables 1 and 2). When soybean cvs. Williams and Essex were inoculated with transcribed RNAs from the VIGS clones together with mild RNA1 transcripts from strain K-G7 (Zhang and Ghabrial 2006), the number of plants infected was very low (Table 1). Because most work published by Zhang and Ghabrial (2006) was done using the severe BPMV RNA1 from strain K-Ho1 on soybean cv. Essex, we compared the infection of BPMV K-G7 RNA2 and RNA1 from either K-Ho1 or K-G7 on cvs. Williams and Essex. There was no major difference in infection of the empty vector with the severe K-Ho1 RNA1 on either cultivar (Table 1). However, there was a major reduction in the number of plants infected using the mild K-G7 RNA1 compared with the severe K-Ho1 RNA1 (Table 1). Because the phenotype of the K-Ho1 RNA1 was severe leaf symptoms and necrosis ( $\mathrm{Gu}$ and Ghabrial 2005), we preferred to use the mild K-G7 RNA1 to obviate interference of symptoms induced by BPMV with those associated with the VIGS phenotype. Therefore, because of the low infection and the requirement of in vitro transcription, we developed an alternative and more efficient DNA-based BPMV VIGS vector technology.

\section{Infectivity of DNA-based BPMV VIGS vectors.}

To facilitate high-throughput VIGS studies, the cDNAs of BPMV genomic RNA1 and RNA2 were placed under control of the CaMV $35 \mathrm{~S}$ promoter (Fig. 1A) to circumvent the need for making in vitro RNA transcripts. In addition, the cloning site in RNA2 was modified to allow multiple host gene sequences to be concatenated for co-silencing (Fig. 1B and C). The use of the CaMV $35 \mathrm{~S}$ promoter allowed, through biolistic delivery, direct introduction of the plasmid DNA of the BPMV VIGS constructs into soybean plants. Both the severe and mild BPMV RNA1s were adapted for use as DNA-based vectors. Three independent tests with empty vectors showed that the DNA-based vector was highly infectious, with close to $100 \%$ of plants infected on either cultivar (Table 1). This contrasts with the low infection by the RNA-based vector, especially for the mild K-G7 RNA1. The high infection makes it feasible to use it as a VIGS vector with the mild K-G7 RNA1 to reduce interference by the severe phenotype associated with strain KHo1 RNA1. Interestingly, we do see a decrease in the number of plants infected with the VIGS constructs compared with the empty vector (Table 1). Nevertheless, satisfactory infection can be readily achieved with the DNA-based vector containing the mild K-G7 RNA1 for which the RNA-based infection was not successful. Additionally, the construct for co-silencing $S g t 1 A+S g t 1 B$ was as infectious as each of the constructs for silencing $S g t 1 A$ or $S g t 1 B$ alone, demonstrating that the vector can be used to co-silence at least two genes with no loss in infection efficiency. We observed little difference among the two soybean cultivars in infection by the different VIGS constructs.

Table 1. Comparison of soybean inoculation with RNA- and DNA-based Bean pod mottle virus (BPMV) virus-induced gene silencing (VIGS) vectors ${ }^{\mathrm{a}}$

\begin{tabular}{|c|c|c|c|c|c|}
\hline Cultivar & RNA1 type & RNA2 type & Target genes & RNA-based inoculation & DNA-based inoculation \\
\hline Williams & K-Ho1 & K-G7 & Empty vector & $8 / 16$ & $15 / 15$ \\
\hline Essex & K-Ho1 & K-G7 & Empty vector & $9 / 16$ & $14 / 15$ \\
\hline Williams & K-G7 & K-G7 & Empty vector & $2 / 16$ & $13 / 15$ \\
\hline Essex & K-G7 & K-G7 & Empty vector & $2 / 16$ & $14 / 15$ \\
\hline Williams & K-G7 & K-G7 & Actin & $1 / 16$ & $10 / 15$ \\
\hline Essex & K-G7 & K-G7 & Actin & $0 / 8$ & $3 / 5$ \\
\hline Williams & K-G7 & K-G7 & Rps6A & $0 / 16$ & $10 / 15$ \\
\hline Essex & K-G7 & K-G7 & Rps6A & $0 / 8$ & $3 / 5$ \\
\hline Williams & K-G7 & K-G7 & Rps13 & $0 / 16$ & $9 / 15$ \\
\hline Essex & K-G7 & K-G7 & Rps 13 & $0 / 8$ & $4 / 5$ \\
\hline Williams & K-G7 & K-G7 & Mpk4A & $1 / 16$ & $10 / 15$ \\
\hline Essex & K-G7 & K-G7 & $M p k 4 \mathrm{~A}$ & $1 / 8$ & $3 / 5$ \\
\hline Williams & K-G7 & K-G7 & $M p k 4 \mathrm{~B}$ & $0 / 16$ & $9 / 15$ \\
\hline Essex & K-G7 & K-G7 & $M p k 4 \mathrm{~B}$ & $0 / 8$ & $2 / 5$ \\
\hline Williams & K-G7 & K-G7 & SgtlA & $0 / 16$ & $9 / 15$ \\
\hline Essex & K-G7 & K-G7 & Sgt1A & $1 / 8$ & $3 / 5$ \\
\hline Williams & K-G7 & K-G7 & Sgt1B & $0 / 16$ & $8 / 15$ \\
\hline Essex & K-G7 & K-G7 & Sgt1B & $0 / 8$ & $2 / 5$ \\
\hline Williams & K-G7 & K-G7 & $S g t 1 \mathrm{~A}+S g t 1 \mathrm{~B}$ & - & $8 / 15$ \\
\hline Essex & K-G7 & K-G7 & Sgt1A+Sgt1B & - & $2 / 5$ \\
\hline
\end{tabular}

a BPMV RNA1 plasmids for RNA-based inoculation are pGG7R1 for mild strain K-G7 and pGHoR1 for severe strain K-Ho1. BPMV RNA1 plasmids for DNA-based inoculation are pG35S-G7R1D for mild strain K-G7 and pG35S-HoR1 for severe strain K-Ho1. The empty RNA2 vector used for RNA-based inoculation is pGG7R2 and for DNA-based inoculation is pG35S-G7R2. A total of $200 \mu \mathrm{l}$ of RNA1 and RNA2 transcripts was used to inoculate eight soybean plants. RNA-based inoculation was repeated once on soybean cv. Williams. One preparation for DNA-based vectors was used to inoculate five soybean plants. Three repetitions were performed on Williams. Both RNA-based and DNA-based inoculations were done when soybean primary leaves were fully expanded (stage VC). BPMV infection was confirmed by double-antibody sandwich enzyme-linked immunosorbent assay. Numbers above and below the diagonal for each cultivar are the numbers of plants infected and the total plants tested, respectively; - = not tested. 


\section{Diverse shoot and root symptoms induced by VIGS constructs.}

One advantage of VIGS is that essential genes that are critically required for plant metabolism can be studied whereas stable mutation may be lethal. With the new DNA-based VIGS vector, we tested the efficacy of silencing soybean homologues of essential genes involved in translation and the cytoskeleton, which would be expected to have severe phenotypes when silenced (Table 1). Silencing two ribosomal proteins, Rps6 and Rps13, induced very severe foliar symptoms (Fig. 2). Silencing of an Actin gene homologue, which is required for the plant cytoskeleton, resulted in severe mosaic, leaf deformation, and stunting (Fig. 2).

The extensive soybean expressed sequence tag (EST) sequence database (Schlueter et al. 2004) allows identification of soybean homologues of Arabidopsis genes previously reported to be involved in defense signaling (Hammond-Kosack and Parker 2003). Two soybean Mpk4 homologues (Mpk4A and $M p k 4 B$ ), which share $57.4 \%$ nucleotide identity and $70.4 \%$ amino acid similarity (data not shown), were identified in soybean in contrast to one in Arabidopsis (Peterson et al. 2000). MPK4 is a negative regulator of Arabidopsis defenses, and loss-of-function mutations result in severely stunted phenotypes. Unlike the mild symptoms induced by silencing $M p k 4 B$ (Fig. 2), silencing $M p k 4 A$ caused severe symptoms, including severe stunting comparable with that on Arabidopsis Mpk4 mutants (Peterson et al. 2000).

Other defense genes are expected to have no phenotype with respect to plant growth and development when silenced. For this purpose, two Arabidopsis Sgt1 gene homologues, also involved in defense signaling (Austin et al. 2002; Tör et al. 2002), were cloned from soybean and inserted into the VIGS vector; after inoculation, infected plants showed mild symptoms similar to the empty vector infected control. To test multiple-gene silencing in soybean, the two Sgtl homologues,
SgtlA and Sgt1B were concatenated into one single construct. Symptoms at 21 days after inoculation showed that the Sgt1A/SgtlB construct induced mild symptoms similar to those induced by $S g t l A$ or $S g t 1 B$ single infection (Fig. 2).

To investigate the effect of the BPMV VIGS vector on silencing endogenous genes in soybean roots, root length of plants infected by the above constructs was measured. One week after

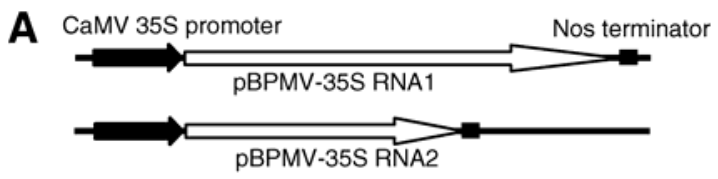

B

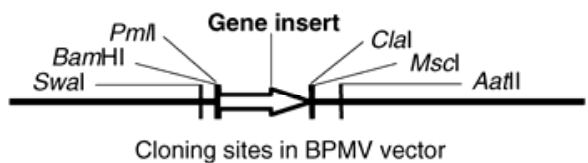

C Cloning multiple gene fragments into BPMV RNA2

Construct a: Notl -- BamHI PmA- gene A - Clal MsCl Construct b: Notl -- BamHI Pm/ - gene B - Clal Mscl

Multiple gene fragments silencing :

Step 1: Construct a supplies backbone and is cut with Notl and PmI. Step 2: Construct b supplies gene B insert and is cut with Notl and MsC Step 3: Gene B digested insert is put into digested construct a to yield: Construct c: Notl-BamHI PmI-gene B-Clal AsetfmH gene A-Clal Msc

Fig. 1. Schematic representation of the Cauliflower mosaic virus (CaMV) $35 \mathrm{~S}$ promoter driven Bean pod mottle virus (BPMV) virus-induced gene silencing (VIGS) vector. A, BPMV genomic RNA1 and RNA2 are expressed under control of the CaMV $35 \mathrm{~S}$ promoter and Nos terminator. pBPMV-35S RNA2 is used for insertion of foreign gene sequences. B, Cloning site for insertion of foreign sequences into BPMV RNA2. C, Cloning strategies for inserting single and multiple genes into the RNA2 VIGS vector. $M s c \mathrm{I}$ and $P m l \mathrm{I}$ are compatible and $M s c \mathrm{I} / P m l \mathrm{I}$ sites destroyed in construct c.

Table 2. Soybean gene fragments cloned into the Bean pod mottle virus vector and resulting phenotype in infected plants

\begin{tabular}{|c|c|c|c|}
\hline Arabidopsis gene type ${ }^{a}$ & Accession number ${ }^{b}$ & Primer sequences $^{c}$ & Phenotype $^{d}$ \\
\hline \multirow[t]{4}{*}{$\operatorname{Actin}(312 \mathrm{bp})$} & TC204141 & VIGS-F: CGCGGATCCCACGTGGCTGGATTTGCTGGTGATGA & $\mathrm{S}$ \\
\hline & $\ldots$ & VIGS-R: ACCCATCGATTCATGGCAGGCACATTGAAGGT & $\ldots$ \\
\hline & $\ldots$ & Probe-F: CGCGGATCCGCCAGCTATCGTTCATCGGA & $\ldots$ \\
\hline & $\ldots$ & Probe-R: CGCGGATCCCAATCAGAACCTACAAAGTTCCA & $\ldots$ \\
\hline \multirow[t]{4}{*}{ Rps6A (297 bp) } & TC205856 & VIGS-F: CGCGGATCCCACGTGGCGAATCCCACCACTGGA & $\mathrm{S}$ \\
\hline & $\ldots$ & VIGS-R: ACCCATCGATTGCACCCACGAACAGACTTTC & $\ldots$ \\
\hline & $\ldots$ & Probe-F: CGCGGATCCCCTCTCTGTTTTGAACTTGGT & $\ldots$ \\
\hline & $\ldots$ & Probe-R: CGCGGATCCCCTCCGATTTGGCCTTGGCA & $\ldots$ \\
\hline \multirow[t]{4}{*}{ Rps13 (333 bp) } & TC225449 & VIGS-F: CGCGGATCCCACGTGCGTATGCACAGCCGCGGT & $\mathrm{S}$ \\
\hline & $\ldots$ & VIGS-R: ACCCATCGATTGGAGTCCTTGTCCTTCCTGT & $\ldots$ \\
\hline & $\ldots$ & Probe-F: CGCGGATCCGAGAGCAGAATCCACCGACT & $\ldots$ \\
\hline & $\ldots$ & Probe-R: CGCGGATCCCCCAGTCAAACCATAGGTTCT & $\ldots$ \\
\hline \multirow[t]{2}{*}{$M p k 4 A(297 \mathrm{bp})$} & TC226687 & VIGS-F: CGCGGATCCCACGTGGCTGCTGTAAATGCAGAGACAGGT & $\mathrm{S}$ \\
\hline & $\ldots$ & VIGS-R: ACCCATCGATTTTTGAGCCCTCGTAACAATTGATA & $\ldots$ \\
\hline \multirow[t]{4}{*}{$M p k 4 B(306 \mathrm{bp})$} & TC216864 & VIGS-F: CGCGGATCCCACGTGGAGAAGGTTGCCATCAAGAAGAT & M \\
\hline & $\ldots$ & VIGS-R: ACCCATCGATTAAGAATGTTAGCGGAGTGAAGAT & $\ldots$ \\
\hline & $\ldots$ & Probe-F: CGCGGATCCTCGTGACTTGAAACCTGGGA & $\ldots$ \\
\hline & $\ldots$ & Probe-R: CGCGGATCCGTAGGAGCTCTGGTGCCCTA & $\ldots$ \\
\hline \multirow[t]{4}{*}{ Sgt1A (306 bp) } & TC203662 & VIGS-F: CGC $\underline{G G A T C C C A C G T G G T T C C T G G C C A A G A T G C C T A T}$ & M \\
\hline & $\ldots$ & VIGS-R: ACCCATCGATTAGCAGCATCACCATCAAGCTTT & $\ldots$ \\
\hline & $\ldots$ & Probe-F: CGCGGATCCTACCAAAATGCAGATGAGGACA & $\ldots$ \\
\hline & $\ldots$ & Probe-R: CGCGGATCCCCACTTCCACAGCAAAAGCA & $\ldots$ \\
\hline \multirow[t]{4}{*}{$\operatorname{Sgt1B}(306 \mathrm{bp})$} & TC206509 & VIGS-F: CGC $\underline{G A T C C C A C G T G T C C G A T C T C G A A G C A A A A G C C A}$ & $\ldots$ \\
\hline & $\ldots$ & VIGS-R: ACCCATCGATTGTCTGGAGACAATGATGCACCCA & $\ldots$ \\
\hline & $\ldots$ & Probe-F: CGCGGATCCAGGTTTGCTACTTTGATCAAAGA & M \\
\hline & $\ldots$ & Probe-R: CGCGGATCCCACCGAAGTCAACAGTAATGCT & $\ldots$ \\
\hline
\end{tabular}

${ }^{a}$ Name of gene used for virus-induced gene silencing (VIGS) constructs. The target gene fragment size in base pairs (bp) is given in parentheses.

${ }^{b}$ Accession numbers are the The Institute for Genomic Research tentative contig (TC) numbers of genes selected for VIGS.

c Primer sequences used for polymerase chain reaction amplification of gene fragments are designated as VIGS-F and VIGS-R for forward and for reverse primers, respectively. Forward or reverse primers used for preparing probes are designated as Probe-F and Probe-R, respectively.

d Soybean phenotype associated with silencing of designated soybean genes on soybean cv. Williams. Abbreviations: M = mild symptoms similar to emptyvector control and $\mathrm{S}=$ severe symptoms relative to empty-vector control. 
biolistic inoculation of the primary leaves of soybean plants (stage VC), soybean roots were trimmed to 1 in. and maintained in deeper pots in the growth chamber under standard growth conditions. Four weeks after inoculation, soybean roots from each of five plants per construct were rinsed and photographed (Fig. 3A). Because the severe symptoms induced by $M p k 4 A$ caused death of the plants at 4 weeks postinoculation, they were not included. As shown (Fig. 3), there was no significant root length difference between the Mock, Vector-alone, and plants infected by the four constructs $M p k 4 B, S g t 1 A, S g t 1 B$, and $S g t 1 A / B$. However, significant root length differences were observed between the empty-vector-infected plants and plants infected by the three constructs Rps6, Rps 13, and Actin that are involved in translation and cytoskeleton (Fig. 3) ( $t$ test, $P<$ $0.001)$. Interestingly, the three constructs that induced severely stunted root growth also induced severe foliar symptoms (Fig. 2 ), indicating the essential role of these genes in protein translation and the cytoskeleton in root and leaf cells.

\section{Quantification of gene expression.}

To confirm that the observed soybean phenotypes associated with various VIGS constructs involve silencing, expression of the target genes was evaluated both at the mRNA level and protein level. Initially, potential changes in the mRNA levels of target genes were examined by Northern blot analysis of total RNAs extracted from leaves of infected plants. Six sets of
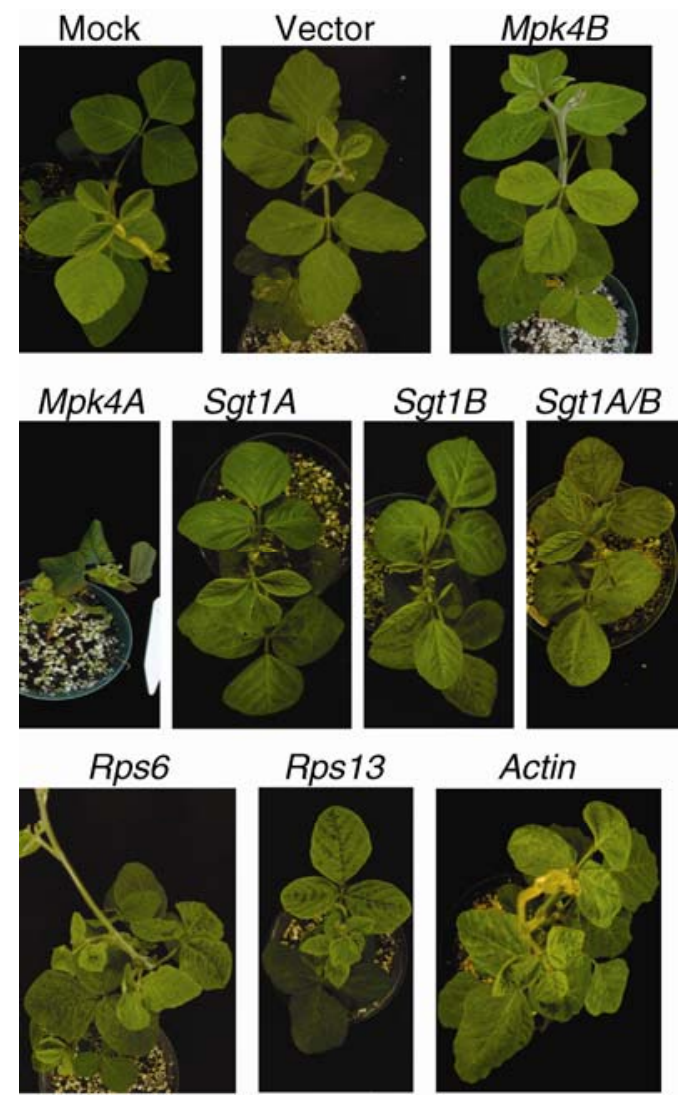

Fig. 2. Diverse shoot phenotypes on the Williams soybean cultivar induced by Bean pod mottle virus (BPMV) virus-induced gene silencing (VIGS) of soybean genes. Plants were photographed at 3 weeks postinoculation. Silencing of $M p k 4 A$ induced very severe symptoms, including stunting and deformation. Severe phenotypes were also induced by silencing of two translation components, Rps6 and Rps13, as well as the cytoskeleton component Actin. Mock $=$ mock-inoculated control. Vector $=$ empty-vector control inoculated with pBPMV-35SG7R1D and pBPMV-35SG7R2. All plants were maintained in a growth chamber at $20^{\circ} \mathrm{C}$ with a photoperiod of $16 \mathrm{~h}$. primers (Table 2, Probe-F and Probe-R) were used to amplify a region of the targeted genes for hybridization that was different from the target gene fragments carried in the VIGS constructs. Total RNAs (15 $\mu \mathrm{g}$ each) were loaded in each lane and equal loading was confirmed by ethidium bromide staining (Fig. 4A). Based upon band intensity relative to the empty vector control (pG35SG7R2) in each blot, reduction in gene expression ranged from 42 to $72 \%$. Symptom severity was not associated with reduction in gene expression because constructs exhibiting a severe phenotype ranged from $42 \%$ for Rps 6 to 64 and $67 \%$ for Rps 13 and Actin, respectively. Among VIGS constructs exhibiting a mild phenotype, reduction was 62,69 , and $72 \%$ for $S g 1 A, S g l B$, and $M p k 4 B$, respectively. Multiple gene silencing, demonstrated by the concatamer construct $S g 1 A / B$, reduced gene expression of $S g 1 A$ and $S g 1 B$ by 51 and $57 \%$, respectively, which was lower than the reduction of gene expression levels of 62 and $69 \%$ induced by the single gene constructs of the same genes (Fig. 4).

Ribosomal protein S6 (Rps6) is a highly conserved component of cytosolic ribosomes (Barakat et al. 2001). Soybean RPS6 has 90\% amino acid identity with Arabidopsis RPS6 (GenBank accession number Y14052) and 92\% with maize RPS6 (GenBank accession number NM_001112074). We used antisera of maize RPS6 for a Western blot analysis of RPS6 accumulation in leaves from empty BPMV vector-infected plants or Rps6-silenced plants. For comparison, $15 \mu \mathrm{g}$ of total protein was loaded per lane and equal loading was confirmed by Coomassie blue staining (Fig. 5). By quantifying the band intensity of the blot, RPS6 was reduced $40 \%$ compared with the
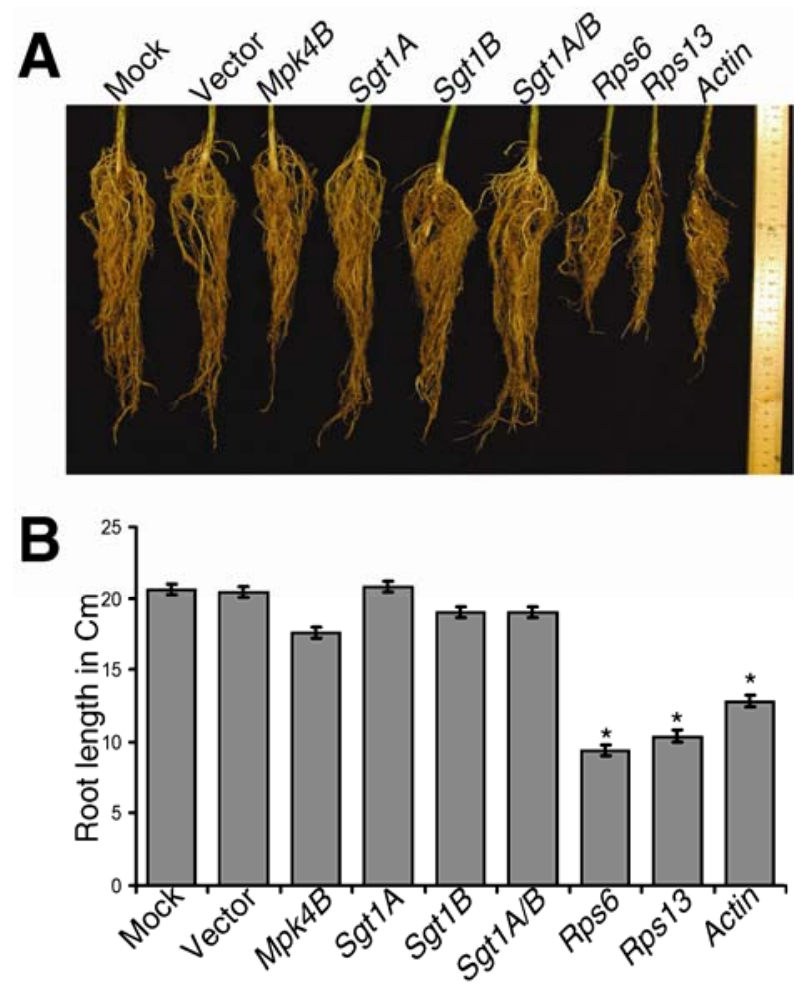

Fig. 3. Root phenotypes on the Williams soybean cultivar induced by Bean pod mottle virus (BPMV) virus-induced gene silencing (VIGS) of soybean genes. A, Effect of silencing essential genes on root growth. Roots were photographed at 4 weeks postinoculation. The names of targeted genes were labeled. Note the difference in root length for plants infected with the three VIGS constructs silencing translation components and cytoskeleton component Actin compared with the other treatments. B, Mean value of root length with standard deviation (bars) for each treatment; * indicates that significant differences $(t$ test, $P<0.001)$ were found between the treatments compared with the Mock and Vector controls. 
empty-vector-infected control (Fig. 5). This protein level reduction is consistent with the mRNA transcript reduction of $42 \%$.

\section{Identification of host genes involved in SMV infection in compatible interaction.}

In order to test the effect of silencing on gene function, BPMV VIGS construct-infected Williams plants, susceptible to both BPMV and SMV, were screened for the effect of silencing on subsequent inoculation by a $\beta$-glucuronidase (GUS)-tagged SMV infectious clone (SMV-N-GUS) (Wang et al. 2006). Three weeks after BPMV inoculation and confirmation of infection by double-antibody sandwich enzyme-linked immunosorbent assay (DAS-ELISA), detached leaves from the empty BPMV vector
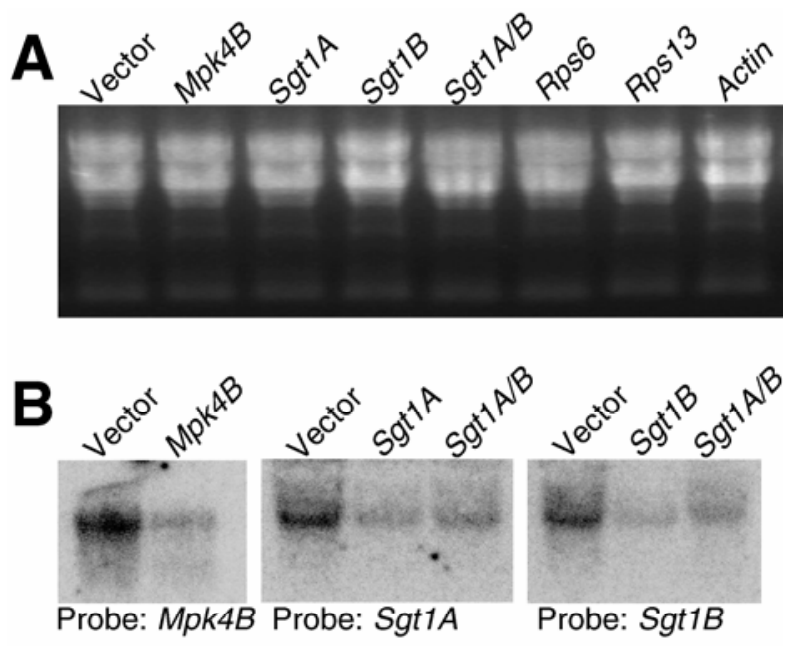

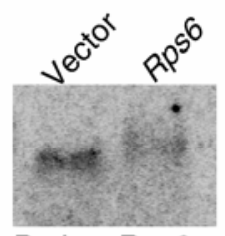

Probe: Rps6

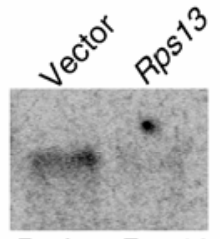

Probe: $R p s 13$

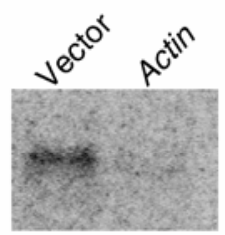

Probe: Actin

\section{C}

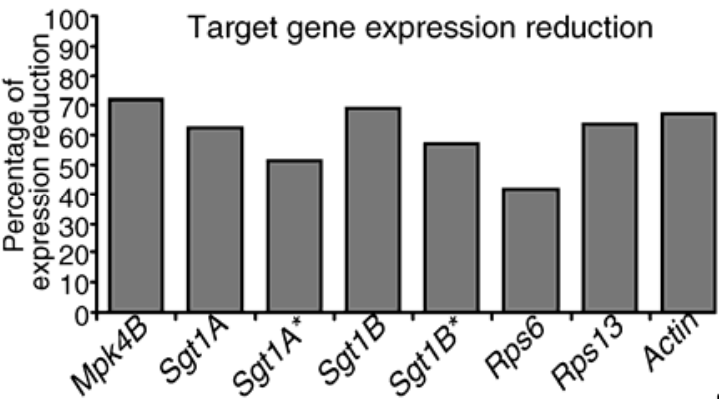

Fig. 4. Changes in the mRNA levels of virus-induced gene silencing (VIGS) target genes. Northern blot analysis was used to assess the mRNA transcript level of each target gene. A, Equivalent RNA loading was assessed by ethidium bromide staining of total RNA extracted from leaves of infected plants by Bean pod mottle virus (BPMV) VIGS constructs. B, Northern blot analysis using probes prepared for each construct. The probe used for each blot is indicated below the corresponding autoradiogram. $\mathbf{C}$, Percentage reduction of the mRNA transcript levels of each target gene compared with their levels in nonsilenced vector control plants. The relative reduction of the mRNA level of each target gene was calculated by subtracting the radioactivity signal of the VIGS treatments from the signal for the nonsilenced vector control and then dividing the difference by the signal of the nonsilenced vector control. The value of the empty-vector-infected plants is $100 \%$ in each gel blot; * indicates the reduction is from the concatemer construct $\operatorname{Sgt} 1 \mathrm{~A} / \mathrm{B}$.
(pG35SG7R2) or from BPMV VIGS construct-infected plants were biolistically inoculated with SMV-N-GUS DNA. At 5 days post SMV-GUS inoculation, a GUS histochemical assay was performed to test for the presence of SMV infection foci. Data were compiled from at least three independent tests for each construct with a total of at least 15 leaves to evaluate VIGS of the selected soybean genes on SMV infection. The Mpk4A construct was not tested because its silencing resulted in severely stunted plants and few leaves were available for the test. For most constructs, including $M p k 4 B$, Sgt1A, Sgt1B, Rps6, and Rps13, there was no discernable difference in the number of SMV infection foci between the empty vector and the VIGS constructs infected plants (data not shown). However, silencing of the soybean Actin gene reduced SMV-N-GUS foci by more than 20-fold when Actin was silenced (Fig. 6). Significant differences occurred between both the Mock and Actin $(t=4.55, P<$ $0.0001)$ as well as the vector and Actin $(t=2.37, P=0.0221)$ treatments. The difference was not significant $(t=1.73, P=$ 0.0902) between the Mock and the Vector treatments.

\section{DISCUSSION}

\section{The DNA-based BPMV VIGS vector is effective} in soybean.

Two RNA transcription and inoculation-based vectors were recently developed for soybean VIGS (Nagamatsu et al. 2007; Zhang and Ghabrial 2006). Following Zhang and Ghabrial's report, the RNA-based BPMV vector was further used to silence two stearoyl-acyl carrier protein-desaturase genes (Kachroo et al. 2008). However, in both BPMV VIGS studies, transcripts from the severe BPMV K-Ho1 RNA1 were used for inoculation because of greater infection efficiency relative to inoculation with transcripts from the mild BPMV K-G7 (Kachroo et al. 2008; Zhang and Ghabrial 2006).

Although BPMV RNA2 was engineered to carry foreign gene sequences, BPMV RNA1 was shown to encode the pathogenicity component that determines foliar symptom severity (Gu and Ghabrial 2005). BPMV strain K-Ho1 RNA1 induces severe symptoms but strain K-G7 RNA1 only causes

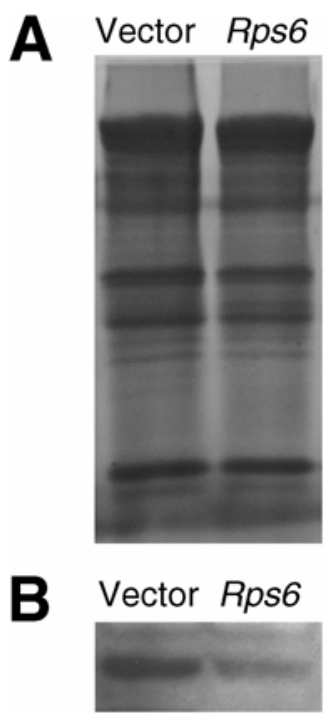

Fig. 5. Immunoblot analysis of RPS6 protein levels in nonsilenced and Rps6-silenced soybean plants. A, Levels of protein loading were assessed by sodium dodecyl sulfate polyacrylamide gel electrophoresis and Coomassie blue staining of the proteins tested. B, Western blot analysis using a maize polyclonal RPS6 antiserum. Total proteins $(15 \mu \mathrm{g})$ were extracted from soybean plants at 21 days postinoculation with the following constructs: Vector, empty-vector control. 
mild symptoms. Even though severe infection by strain K-Hol RNA1 enhances foreign gene expression by the BPMV vector, the mild infection by K-G7 RNA1 is ideal for VIGS studies, because there is less potential for viral symptoms to confound the silencing phenotypes.

This study showed that infectivity of K-G7 RNA1 was relatively low compared with transcripts from K-Ho1 RNA1 when combined with RNA2 from K-G7 and used to inoculate two soybean cultivars. Experiments were conducted in parallel using the same reagents and freshly prepared RNA transcripts for inoculation. Further, no difference was observed in integrity of the transcripts on $1 \%$ agarose gels (data not shown). Although increased repetition is feasible, it is not cost effective or suitable for studies requiring high-throughput analyses. Consequently, an alternative approach was used to develop a DNA-based BPMV vector. Comparison of the DNA-based vector with the RNA-based vector showed greater infectivity of the DNA-based vector. Further, when the mild K-G7 RNA1 was used with K-G7 RNA2, infectivity was equivalent to use of the severe K-Ho1 RNA1. Therefore, the K-G7 RNA1 was used in experiments in which a variety of soybean gene homo-

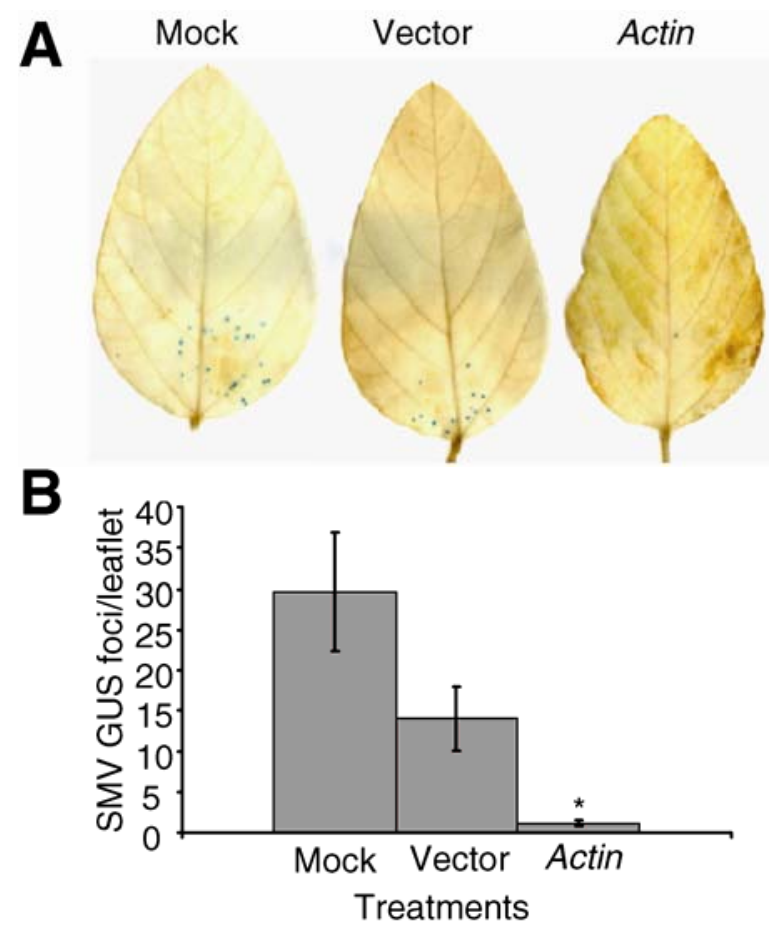

Fig. 6. Bean pod mottle virus (BPMV) virus-induced gene silencing (VIGS) of an Actin gene reduced Soybean mosaic virus (SMV) virus accumulation. A, Scanned leaves of the Williams soybean cultivar with $\beta$-glucuronidase (GUS)-tagged SMV infectious clone (SMV-N-GUS) infection foci. Plants were first inoculated with the BPMV VIGS constructs indicated above each picture. Three weeks postinoculation with VIGS constructs and confirmation by double-antibody sandwich enzyme-linked immunosorbent assay, upper soybean leaves were detached and biolistically inoculated with SMV-N-GUS. Following SMV inoculation, the detached leaves were kept in a growth chamber for 5 days before staining and scanning. Mock = soybean plant was first inoculated with leaf sap from healthy plants and then inoculated with SMV-N-GUS. Vector = soybean plant was first inoculated with empty BPMV vector pBPMV-35SG7R2 and then challenged with SMV-N-GUS. Mock and Vector treatments showed typical SMV-GUS infection foci. Actin = BPMV construct silencing cytoskeleton actin component reduced SMV-N-GUS infection foci. B, Mean number of foci/leaflet plus standard deviation (bars) for each treatment; * $=$ significant differences $(P<0.05, t$ test $)$ were found between Mock and Actin treatments as well as between Vector and Actin treatments. Differences in foci number between Mock and Vector treatments were not significant at $P<0.05$. logues were inserted into K-G7 RNA2 and used in direct comparison of infectivity of the RNA- and DNA-based vectors. In all cases, infectivity of the DNA-based vector was greater and, in a majority of the trials (Rps6A, Rps 13, Mpk4B, and Sgt1B), no infectivity was obtained with the RNA-based vector in the number of repetitions attempted. The DNA-based vector, using the mild K-G7 RNA1 with reduced symptom interference, has been convenient to handle; for example, the K-G7 RNA1 plasmid DNAs prepared and stored at $-20^{\circ} \mathrm{C}$ for more than 1 year have shown no reduction in infectivity after biolistic inoculation. This provides convenience that enables large-scale studies and distribution of the vector to multiple collaborators.

The DNA-based BPMV vector was also designed to silence multiple genes using a single construct to make possible simultaneous testing of different combinations of genes or homologues in soybean that have genetic redundancy (Blanc and Wolfe 2004; Schlueter et al. 2004; Shoemaker et al. 1996) or function in parallel signaling pathways (Lawrence and Pikaard 2003). We did not test the possibility of using two RNA2 VIGS constructs for simultaneous silencing of two genes because of the potential complexity of co-infection by two independent constructs in a single plant (Fraser 1998; Roossinck 2005). Instead, the vector described here is designed for multiple-gene silencing with a single construct and is expandable by concatemerization (Fig. 1). Because it is necessary to clone the foreign gene insert in frame with the RNA2 polyprotein, the vector was designed so that fusion of foreign inserts from two constructs does not disrupt translation (Fig. 1). This concept was validated by the simultaneous silencing of Sgt1A/SgtlB (Fig. 4). Zhang and Ghabrial (2006) reported that up to $1.4 \mathrm{~kb}$ of foreign sequence can be inserted into BPMV RNA2 and, therefore, at least four 300-bp gene fragments can theoretically be inserted into one construct for silencing.

A potential rate-limiting step in the use of VIGS for highthroughput analyses of genes is the necessity to clone and insert multiple gene fragments. Recently, we modified the DNA-based BPMV vector with topoisomerase for high-throughput cloning, and infectivity tests showed that there is no observable difference in infection efficiency between the topoisomerase-modified vector and the parent vector (unpublished data).

\section{BPMV as an effective gene-silencing vector in soybean shoot and root systems.}

The BPMV vector was used to examine gene silencing in both soybean shoots and roots. As expected, very mild leaf symptoms occurred when K-G7 RNA1 was used with the empty vector RNA2. Similar mild leaf phenotypes also occurred with several gene inserts; however, enhanced foliar phenotypes did occur with soybean homologues of genes associated with plant defense, the cytoskeleton, and translation. For example, a severe phenotype was induced by silencing soybean $M p k 4 A$ and this phenotype is similar to that associated with $M p k 4$ loss-of-function in Arabidopsis (Peterson et al. 2000). Interestingly, BLAST search of the translated soybean EST database, using the Arabidopsis Mpk4 protein sequence, identified two soybean homologues, $M p k 4 A$ and $M p k 4 B$, that share $57.4 \%$ nucleotide identity. $M p k 4 B$ is expressed in soybean (Fig. 4). Silencing $M p k 4 B$ induced a mild phenotype. The different phenotypes associated with silencing $M p k 4 A$ and $M p k 4 B$ suggested that similar functions were shared between Arabidopsis $M p k 4$ and soybean $M p k 4 A$ but not soybean $M p k 4 B$, demonstrating the usefulness of the new DNA-based VIGS vector for soybean gene function validation.

Soybean homologues of Arabidopsis defense genes Sgt1A and Sgt1B (Austin et al. 2002; Tör et al. 2002) induced mild phenotypes in soybean upon silencing. The concatamer construct $S g t 1 A / B$ did not significantly enhance the phenotype al- 
though both genes were successfully silenced. Thus, the results support the suggestion that repression of gene expression and plant phenotype are not necessarily closely associated in soybeans.

As in shoots, the application of a reverse genetics tool will be important for potential study of water and nutrient uptake as well as microbial- and nematode-root interactions (Constantin et al. 2008; Rosso et al. 2005; Ryu et al. 2004). Therefore, the effect of silencing the array of soybean genes on root development was measured. The silencing of three essential genes was associated with stunted root development, demonstrating the usefulness of the vector for the study of gene function in soybean roots. Because the BPMV vector is inherently designed for foreign gene expression (Zhang and Ghabrial 2006), the new high efficiency vector developed here may also be used for foreign gene expression to test gene function in a variety of applications in soybean roots as well as shoots.

Reverse-transcription polymerase chain reaction (RT-PCR) has been successfully used to demonstrate silencing of target genes in numerous plant species (Burch-Smith et al. 2004; Liu et al. 2002; Oh et al. 2008; Xu et al. 2007). However, RT-PCR did not consistently reveal reduced gene expression in soybean (data not shown). Alternatively, similar to a Cucumber mosaic virus (CMV) VIGS study in soybean (Nagamatsu et al. 2007), a RNA Northern blot analysis was used to evaluate soybean gene expression level. A consistent reduction of mRNA transcript levels corresponding to all the genes targeted by VIGS was observed. The reduction in gene expression, ranging from 42 to $72 \%$, is close to the $53 \%$ reduction reported for a soybean chalcone synthase gene using a CMV VIGS vector (Nagamatsu et al. 2007). The mosaic bleaching phenotype resulting from silencing PDS as reported by Zhang and Ghabrial (2006) is also suggestive of incomplete silencing. However, the previous study did not examine the potential that a differential level of silencing occurred in the bleached and nonbleached areas of soybean leaves. Further, our tests showed that reduction in gene expression is independent of phenotypes we observed (Figs. 2 and 4).

Because Rps6 is highly conserved among soybean and maize, antisera against maize Rps 6 was used to confirm reduced gene expression at the protein level. Northern blots revealed a $42 \%$ reduction in the Rps6 mRNA and a corresponding $40 \%$ reduction in RPS6 protein level by Western blot. Recently, Brodersen and associates (2008) reported that siRNA, the key molecule for VIGS, has a widespread translational repression effect on gene expression. Interestingly, we did not observe a further reduction in RPS6 protein based on the comparison of the Northern and Western blots.

\section{Functional analysis of the soybean Actin gene in SMV infection.}

Actin is a ubiquitous protein found in eukaryotic cells associated with the plant cytoskeleton which is primarily composed of microtubules and microfilaments that interact and are involved in diverse plant responses (Rodriguez et al. 2003; Wasteneys and Yang 2004). Previously, the association of microtubules with the movement protein of TMV was shown to be important for TMV virus movement (Boevink and Oparka 2005; McLean et al. 1995). Further, silencing of the Actin gene reduced virus movement of TMV, a member of Tobamovirus (Liu et al. 2005). Our data demonstrate that silencing of the Actin gene is also associated with a reduced infection of SMV, a member of Potyvirus. This suggests that the Actin gene may have a broader role in plant virus infection. In addition, this result demonstrates the utility of the BPMV VIGS vector as an effective tool for identifying soybean genes with roles in hostvirus interactions.

\section{MATERIALS AND METHODS}

\section{Virus strains, inoculation, and BPMV virus detection.}

BPMV strains K-G7 and K-Ho1 and the clones used in this study have been previously described (Zhang and Ghabrial 2006). Seed of the two soybean cvs. Essex and Williams used in this study were harvested from greenhouse-grown plants previously indexed for the absence of BPMV and SMV. All BPMV viral infection was verified by DAS-ELISA (Bradshaw et al. 2007).

Synthesis of in vitro RNA transcripts of BPMV was previously described by Zhang and Ghabrial (2006). DNA-based BPMV constructs were biolistically introduced into the primary leaves (VC stage) of 12-day-old soybean seedlings by a single particle bombardment as described (Sanford et al. 1993) using a Biolistic PDS-1000/He system (Bio-Rad Laboratories, Hercules, CA, U.S.A.), $1.0 \mu \mathrm{m}$ gold particles, and 1,100-psi rupture disks at a distance of $6 \mathrm{~cm}$. The leaves were supported by a plastic disk that is notched so it can be centered under leaves without damaging the stem. The leaves were held in place and flat on the disk by a coarse metal mesh. Following bombardment, plants were maintained in the greenhouse or growth chamber at $20^{\circ} \mathrm{C}$ with a photoperiod of $16 \mathrm{~h}$.

\section{In vitro RNA transcription-based VIGS constructs.}

The primer pairs (VIGS-F for forward and VIGS-R for reverse) (Table 2) were used for PCR amplification using oligo $(\mathrm{dT})_{20}$ cDNA template, and the PCR products were cloned into pGEM-T Easy (Promega Corp., Madison, WI, U.S.A.). Inserted sequences were confirmed by sequencing using M13 forward and reverse primers. For directional insertion of the soybean gene fragments into the BPMV VIGS vector, a BamHI and a ClaI restriction site was included in the forward and reverse primers, respectively (Table 2). An extra restriction site for $P m l I$ was included in the forward primer for future concatemer construction. The insert in the pGEM-T Easy vector was digested with Bam HI and ClaI sequentially and ligated into similarly treated empty-vector pGG7R2-V. The resulting constructs were sequenced and tested for infectivity with either K-Ho1 or K-G7 RNA1 transcripts by in vitro transcript inoculation as described by Zhang and Ghabrial (2006).

\section{Vector modification and construction of CaMV 35S promoter-driven BPMV RNA1.}

Unless otherwise stated, all plasmids were propagated in ElectroMax DH5 $\alpha$-E cells (Invitrogen, Carlsbad, CA, U.S.A.) and purified using the QiaPrep spin miniprep kit (Qiagen, Valencia, CA, U.S.A.). All nucleotide sequencing was done using the Big Dye Terminator DNA Sequencing Kit (Applied Biosystems, Foster City, CA, U.S.A.) and the ABI Prism 310 genetic analyzer. Sequence analysis was performed using the Vector NTI program (Invitrogen).

The fragment containing transcription elements from pBR322-35S (Wang et al. 2006) was released by digestion with EcoRI and NotI and put into the same sites in digested pGEM-11zf(+) vector (Promega Corp.) to generate the pGEM$35 \mathrm{~S}$ vector. pGEM-35S was digested with $M s c \mathrm{I}$ and EcoRI and then Klenow treated and self ligated to remove $M s c \mathrm{I}$ from the pGEM-11zf(+) vector backbone. The resultant vector was named pGEM-35S-M1.

BPMV RNA1 cDNA clone pGG7R1 (Zhang and Ghabrial 2006) was used as a template to amplify the $5^{\prime}$ end of RNA1 with primer pair BP-5endF and BP-G7R1-1064R (Table 3) using Takara PrimeSTAR HS DNA Polymerase following the manufacturer's recommended protocol (TaKaRa Bio Inc., Otsu, Shiga, Japan). The PCR product was treated with T4 DNA 
kinase and ligated into StuI-digested pGEM-35S to generate construct pG35S-G7R1A. Insertion was confirmed by sequencing with primers 35S-Seq, BP-R1-235F, BP-R1-688F, and NosRev (Table 3). BPMV RNA1 3' end was amplified from pGG7R1 with primer pair BP-G7R1-5708Cla and BP-R1-3Cla. The $3^{\prime}$ end PCR product was digested with $C l a \mathrm{I}$ and inserted into ClaI-digested clone pG35S-G7R1A to generate construct pG35S-G7R1B. Insertion direction and sequence were confirmed by sequencing with primer Nos-Rev. Clone pGG7R1 was digested with NheI and Eco47III and the released $4.3 \mathrm{~kb}$ was gel purified using the QIAquick gel extraction kit (Qiagen). This fragment was ligated into pG35S-G7R1B digested with the same enzymes to generate construct pG35S-G7R1C. Clone pGG7R1 was digested with $S w a I$ and $X h o I$ and ligated into pG35S-G7R1C digested with the same enzymes to generate construct pG35S-G7R1D, which is the full-length infectious clone used as the RNA1 component for all experiments. BPMV RNA1 cDNA clone pGHoR1 (Zhang and Ghabrial 2006) was used as template to amplify K-Ho1 RNA1 with primer pair BP5endF and BP-R1-3Cla (Table 3). The PCR product was digested with $S w a \mathrm{I}$ and $\mathrm{ClaI}$ and the 5.7-kb fragment was ligated into pG35S-G7R1D digested with the same enzymes to yield full-length infectious clone pG35S-HoR1.

CaMV 35S promoter driven BPMV RNA2 VIGS constructs. pGG7R2 and pGG7R2-V (Zhang and Ghabrial 2006) were used as template with primer pair BP-5end-F and BP-R2-3Cla (Table 3) for PCR amplification. The PCR products were treated with T4 DNA kinase and digested with $C l a \mathrm{I}$ and ligated into StuI and ClaI digested pGEM-35S-M1 vector to generate clones pG35S-G7R2 and pG35S-G7R2V1. Insert orientation was confirmed by sequencing with primer $35-\mathrm{Seq}$ (Table 3) and the entire genomic BPMV G7 RNA2 insertion was sequenced with primers listed in Table 3. Infectivity of pG35S-G7R2 was tested with pG35S-G7R1D or pG35S-HoR1. pG35S-G7R2V1 was partially digested with $\mathrm{ClaI}$ and treated with Klenow polymerase large fragment and self ligated to remove the $3^{\prime}$ end ClaI site to yield pG35S-G7R2V2. Previously described pGEM-T Easy vector clones containing soybean gene inserts were digested sequentially with BamHI and ClaI. The BamHI site is in frame with the viral polyprotein, and it is italicized and underlined in the forward primers for each gene listed in Table 2. The gelpurified inserts were ligated into similarly treated pG35SG7R2V2, and the resulting constructs were sequenced and tested for infectivity by particle bombardment with pG35SG7R1D or PG35S-HoR1.

pG35S-Sgt1A was digested with NotI and PmlI (Fig. 1C, construct a) and the 5-kb fragment was gel recovered. pG35SSgt1B was digested with NotI and MscI (Fig. 1C, construct b) and the $3-\mathrm{kb}$ fragment was gel recovered and ligated with the 5-kb pG35S-Sgt1A fragment to yield pG35S-Sgt1A/B (Fig. $1 \mathrm{C}$, construct $\mathrm{c})$.

\section{RNA extraction and Northern hybridization analysis.}

Total RNA was extracted from soybean leaves by the Trizol method (Invitrogen). First-strand cDNA was synthesized using $2 \mu \mathrm{g}$ of total RNA, $0.5 \mu \mathrm{g}$ of oligo(dT) $)_{20}$ primer, $1 \mu \mathrm{l}$ of 10 $\mathrm{mM} \mathrm{dNTP}$ and Superscript III reverse transcriptase (Invitrogen) to a final volume of $20 \mu \mathrm{l}$.

For Northern hybridization analysis, RNA was extracted from soybean leaf samples. Following RNA extraction, hybridization was performed according to the description by Zhang and Ghabrial (2006). The probe for each VIGS target gene was selected from a region of the gene fragment using primers (Probe-F for forward and Probe-R for reverse) listed in Table 2. Probe sequences were amplified with the primer pairs and oligo(dT) $)_{20}$ cDNA as template and the PCR products were digested with BamHI and cloned into BamHI-digested and dephosphorylated pGEM-11zf(+) vector. The resultant clones were sequenced with M13 primer to confirm the identity of the inserted sequence and used as template with corresponding Probe-F and Probe-R primer pairs for PCR amplification to obtain template DNA for probe preparation. Probes were labeled using the Prime-a-Gene labeling system (Promega Corp.). Northern blot was assessed using ImageQuant v5.2 (Amersham, Piscataway, NJ, U.S.A.).

\section{Protein extraction and Western blot analysis.}

Three weeks after inoculation, fresh leaf (or root) tissues were collected and protein was extracted as described (Moffett et al. 2002). Protein concentration was estimated by the BioRad protein assay method (Bio-Rad Laboratories). Proteins were separated by polyacrylamide gel electrophoresis (PAGE) using $12 \%$ stacking and $15 \%$ resolving polyacrylamide with sodium dodecyl sulfate (SDS) running buffer $(25 \mathrm{mM}$ Tris, $\mathrm{pH}$ $8.3 ; 192 \mathrm{mM}$ glycine; and 1\% SDS), and transferred to polyvinylidene difluoride (PVDF) membrane (Bio-Rad Laboratories). Equal protein loading was estimated by staining a parallel gel with Coomassie blue. The PVDF membranes were incubated in 5\% nonfat dry milk/phosphate-buffered saline-Tween blocking buffer (137 mM NaCl, $2.7 \mathrm{mM} \mathrm{KCl}, 8 \mathrm{mM} \mathrm{Na}_{2} \mathrm{HPO}_{4}, 2$ $\mathrm{mM} \mathrm{KH} \mathrm{KO}_{4}, \mathrm{pH}$ to 7.4 with $\mathrm{HCl}, 0.1 \%$ Tween-20) for $3 \mathrm{~h}$ at room temperature, and then incubated overnight at $4{ }^{\circ} \mathrm{C}$ with antiserum to maize RPS6 diluted 1:1,250 (Williams et al. 2003). This was followed by incubation with goat anti-rabbit immunoglobulin $\mathrm{G}$ conjugated with horseradish peroxidase (1:10,000 dilution; Amersham) for $1 \mathrm{~h}$ at room temperature. The blot images were developed using an enhanced chemilu-

Table 3. Primers for constructing and sequencing DNA-based Bean pod mottle virus virus-induced gene silencing vectors

\begin{tabular}{ll}
\hline Name & \multicolumn{1}{c}{${\text { Sequence }\left(\mathbf{5}^{\prime} \rightarrow \mathbf{3}^{\prime}\right)}^{\prime}$} \\
\hline BP-5endF & TATTAAAATTTTCATAAGATTTGAAATTTTG \\
BP-R1-3Cla & CCATCGATTTTTTTTTTTTTTTTTATATTTAAACAC \\
BP-R1-235F & ATATAGGACTTCGTGTCAGATT \\
BP-R1-688F & TGCATATCATTTTCAGCATTTTGT \\
BP-G7R1-1064R & TCCTAGAGCACAGGAGCTAGCCA \\
BP-G7R1-5708Cla & CCCATCGATCAATAAGAAAATCGGACAGCGCTT \\
BP-R2-3Cla & CCATCGATTTTTTTTTTTTTTTTTAAAATAACACAC \\
BP-G7R2-454F & ACTTGGGCATTGGTGCAAATGT \\
BP-G7R2-948F & ACTTCTTACTGATGGGAAGTTGTA \\
BP-G7R2-1548F & CAAGAGAAAGATTTATTGGAGGGA \\
BP-G7R2-1786F & AAGCTCAAATGGAAACAAATCT \\
BP-G7R2-2133F & TGGAATCCTGCTTGTACAAAAGCA \\
BP-G7R2-2746F & TGGCTGATGGGTGCCCATATT \\
BP-G7R2-3303F & ATGGTTTCGAAATGTGGAGTTCTGA \\
35S-Seq & ACGCACAATCCCACTATC \\
Nos-Rev & AGACCGGCAACAGGATTCA \\
\hline
\end{tabular}


minescence system (Amersham) according to the manufacturer's instructions. Western blot was assessed using ImageQuant v5.2.

\section{ACKNOWLEDGMENTS}

We thank Jeff D. Bradshaw for help with the statistical analysis of root length and actin silencing. This work was funded partly by the Iowa State University Plant Sciences Institute Innovation Research Grants Program, the North Central Soybean Research Program, the Iowa Soybean Association, and Hatch Act and State of Iowa Funds.

\section{LITERATURE CITED}

Austin, M. J., Muskett, P., Kahn, K., Feys, B. J., Jones, J. D., and Parker, J. E. 2002. Regulatory role of SGT1 in early $R$ gene-mediated plant defenses. Science 295:2032-2033.

Barakat, A., Szick-Miranda, K., Chang, I. F., Guyot, R., Blanc, G., Cooke, R., Delseny, M., and Bailey-Serres, J. 2001. The Organization of cytoplasmic ribosomal protein genes in the Arabidopsis genome. Plant Physiol. 127:398-415.

Blanc, G., and Wolfe, K. H. 2004. Widespread paleopolyploidy in model plant species inferred from age distributions of duplicate genes. Plant Cell 16:1667-1678.

Boevink, P., and Oparka, K. J. 2005. Virus-host interactions during movement processes. Plant Physiol. 138:1815-1821.

Bradshaw, J. D., Rice, M. E., and Hill, J. H. 2007. No-choice preference of Cerotoma trifurcata (Coleoptera: Chrysomelidae) to potential host plants of Bean pod mottle virus Comoviridae in Iowa. J. Econ. Entomol. 100:808-814.

Brodersen, P., Sakvarelidze-Achard, L., Bruun-Rasmussen, M., Dunoyer, P., Yamamoto, Y. Y., Sieburth, L., and Voinnet, O. 2008. Widespread translational inhibition by plant miRNAs and siRNAs. Science 320:1185-1190.

Burch-Smith, T. M., Anderson, J. C., Martin, G. B., and Dinesh-Kumar, S. P. 2004. Applications and advantages of virus-induced gene silencing for gene function studies in plants. Plant J. 39:734-746.

Burch-Smith, T. M., Schiff, M., Liu, Y., and Dinesh-Kumar, S. P. 2006. Efficient virus-induced gene silencing in Arabidopsis. Plant Physiol. 142:21-27.

Constantin, G. D., Krath, B. N., MacFarlane, S. A., Nicolaisen, M., Johansen, I. E., and Lund, O. S. 2004. Virus-induced gene silencing as a tool for functional genomics in a legume species. Plant J. 40:622-631.

Constantin, G. D., Gronlund, M., Johansen, I. E., Stougaard, J., and Lund, O. S. 2008. Virus-induced gene silencing (VIGS) as a reverse genetic tool to study development of symbiotic root nodules. Mol. PlantMicrobe Interact. 21:720-727.

Ding, W. X., Schneider, W. L., Chaluvadi, S. R., Rouf, M. A., and Nelson, R. A. 2006. Characterization of a Brome mosaic virus strain and its use as a vector for gene silencing in monocotyledonous hosts. Mol. PlantMicrobe Interact. 19:1229-1239.

Fraser, R. S. S. 1998. Introduction to classical crossprotection. Pages $13-$ 24 in: Methods in Molecular Biology, Plant Virus Protocols, vol. 81. G. D. Foster and S. C. Taylor, eds. Humana Press, Totowa, NJ, U.S.A.

$\mathrm{Gu}, \mathrm{H}$., and Ghabrial, S. A. 2005. The Bean pod mottle virus proteinase cofactor and putative helicase are symptom severity determinants. Virology 333:271-283.

Hammond-Kosack, K. E., and Parker, J. E. 2003. Deciphering plantpathogen communication: Fresh perspectives for molecular resistance breeding. Curr. Opin. Biotechnol. 14:177-193.

Jackson, S. A., Rokhsar, D., Stacey, G., Shoemaker, R. C., Schmutz, J., and Grimwood, J. 2006. Toward a reference sequence of the soybean genome: A multiagency effort. Crop Sci. 46:S55-S61.

Kachroo, A., Fu, D. Q., Havens, W., Navarre, D., Kachroo, P., and Ghabrial, S. A. 2008. An oleic acid-mediated pathway induces constitutive defense signaling and enhanced resistance to multiple pathogens in soybean. Mol. Plant-Microbe Interact. 21:564-575.

Lawrence, R. J., and Pikaard, C. S. 2003. Transgene-induced RNA interference: A strategy for overcoming gene redundancy in polyploids to generate loss-of-function mutations. Plant J. 36:114-121.

Liu, J. Z., Blancaflor, E. B., and Nelson, R. S. 2005. The Tobacco mosaic virus 126-kilodalton protein, a constituent of the virus replication complex, alone or within the complex aligns with and traffics along microfilaments. Plant Physiol. 138:1853-1865.

Liu, Y., Schiff, M., and Dinesh-Kumar, S. P. 2002. Virus-induced gene silencing in tomato. Plant J. 31:777-786.
Lu, R., Malcuit, I., Moffett, P., Ruiz, M. T., Peart, J., Wu, A. J., Rathjen, J. P., Bendahmane, A., Day, L., and Baulcombe, D. C. 2003. High throughput virus-induced gene silencing implicates heat shock protein 90 in plant disease resistance. EMBO (Eur. Mol. Biol. Organ.) J. 22:5690-5699.

McLean, G., Zupan, J., and Zambryskil, P. C. 1995. Tobacco mosaic virus movement protein associates with the cytoskeleton in tobacco cells. Plant Cell 7:2101-2121.

Moffett, P., Farnham, G., Peart, J., and Baulcombe, D. C. 2002. Interaction between domains of a plant NBS-LRR protein in disease resistancerelated cell death. EMBO (Eur. Mol. Biol. Organ.) J. 21:4511-4519.

Nagamatsu, A., Masuta, C., Senda, M., Matsuura, H., Kasai, A., Hong, J. S., Kitamura, K., Abe, J., and Kanazawa, A. 2007. Functional analysis of soybean genes involved in flavonoid biosynthesis by virus-induced gene silencing. Plant Biotechnol. J. 5:778-790.

Oh, S. K., Baek, K. H., Park, J. M., Yi, S. Y., Yu, S. H., Kamoun, S., and Choi, D. 2008. Capsicum annuum WRKY protein CaWRKY1 is a negative regulator of pathogen defense. New Phytol. 177:977-989.

Petersen, M., Brodersen, P., Naested, H., Andreasson, E., Lindhart, U., Johansen, B., Nielsen, H. B., Lacy, M., Austin, M. J., Parker, J. E., Sharma, S. B., Klessig, D. F., Martienssen, R., Mattsson, O., Jensen A. B., and Mundy J. 2000. Arabidopsis MAP kinase 4 negatively regulates systemic acquired resistance. Cell 103:1111-1120.

Rodriguez, O. C., Schaefer, A. W., Mandato, C. A., Forscher, P., Bement, W. M., and Waterman-Storer, C. M. 2003. Conserved microtubule-actin interactions in cell movement and morphogenesis. Nat. Cell Biol. 5:599-609.

Roossinck, M. J. 2005. Symbiosis versus competition in plant virus evolution. Nat. Rev. Microbiol. 3:917-924.

Rosso, M. N., Dubrana, M. P., Cimbolini, N., Jaubert, S., and Abad, P. 2005. Application of RNA interference to root-knot nematode genes encoding esophageal gland proteins. Mol. Plant-Microbe Interact. 18:615620.

Ryu, C. M., Anand, A., Kang, L., and Mysore, K. S. 2004. Agrodrench: A novel and effective agroinoculation method for virus-induced gene silencing in roots and diverse Solanaceous species. Plant J. 40:322-331.

Sanford, J. C., Smith, F. D., and Russell, J. A. 1993. Optimizing the biolistic process for different biological applications. Methods Enzymol. 217:483-509.

Schlueter, J. A., Dixon, P., Granger, C., Grant, D., Clark, L., Doyle, J. J., and Shoemaker, R. C. 2004. Mining EST databases to resolve evolutionary events in major crop species. Genome 47:868-876.

Senthil-Kumar, M., Anand, A., Uppalapati, S. R., and Mysore, K. S. 2008 Virus-induced gene silencing and its applications. CAB Rev. Perspect. Agric. Vet. Sci. Nutr. Nat. Resour. 3:011.

Shoemaker, R. C., Polzin, K., Labate, J., Specht, J., Brummer, E. C., Olson, T., Young, N., Concibido, V., Wilcox, J., Tamulonis, J. P., Kochert, G., and Boerma, H. R. 1996. Genome duplication in soybean Glycine subgenus soja. Genetics 144:329-338.

Stacey, G., Vodkin, L., Parrott, W. A., and Shoemaker, R. C. 2004. National Science Foundation-sponsored workshop report: Draft plan for soybean genomics. Plant Physiol. 135:59-70.

Tör, M., Gordon, P., Cuzick, A., Eulgem, T., Sinapidou, E., Mert-Türk, F., Can, C., Dangl, J. L., and Holub, E. B. 2002. Arabidopsis SGT1b is required for defense signaling conferred by several downy mildew resistance genes. Plant Cell 14:993-1003.

Turnage, M. A., Muangsan, N., Peele, C. G., and Robertson, D. 2002. Geminivirus-based vectors for gene silencing in Arabidopsis. Plant J. 30:107-117.

Wang, L., Eggenberger, A., Hill, J. H., and Bogdanove, A. J. 2006. Pseudomonas syringae effector $a v r B$ confers soybean cultivar-specific avirulence on Soybean mosaic virus adapted for transgene expression but effector avrPto does not. Mol. Plant-Microbe Interact. 19:304-312.

Wasteneys, G. O., and Yang, Z. 2004. The cytoskeleton becomes multidisciplinary. Plant Physiol. 36:3853-3854.

Williams, A. J., Werner-Fraczek, J., Chang, I. F., and Bailey-Serres, J. 2003. Regulated phosphorylation of 40S ribosomal protein S6 in root tips of maize. Plant Physiol. 132:2086-2097.

Xu, Y. P., Zheng, L. P., Xu, Q. F., Wang, C. C., Zhou, X. P., Wu, Z. J., and Cai, X. Z. 2007. Efficiency for gene silencing induction in Nicotiana species by a viral satellite DNA vector. J. Integr. Plant Biol. 49:1726-1733.

Zhang, C., and Ghabrial, S. A. 2006. Development of Bean pod mottle virus-based vectors for stable protein expression and sequence-specific virus-induced gene silencing in soybean. Virology 344:401-411.

\section{AUTHOR-RECOMMENDED INTERNET RESOURCE}

The Institute for Genomic Research website: www.tigr.org 